Gas Electron Multiplier (GEM) application for Time Projection Chamber (TPC) gating

This article has been downloaded from IOPscience. Please scroll down to see the full text article.

2010 JINST 5 P03001

(http://iopscience.iop.org/1748-0221/5/03/P03001)

The Table of Contents and more related content is available

Download details:

IP Address: 137.138.125.144

The article was downloaded on 08/04/2010 at 16:21

Please note that terms and conditions apply. 
$1^{\text {st }}$ International Conference on Micro Pattern Gaseous Detectors, JUNE 12-15, 2009

Kolympari, Crete, Greece

\title{
Gas Electron Multiplier (GEM) application for Time Projection Chamber (TPC) gating
}

\section{G. Croci, ${ }^{a, b}$ M. Alfonsi, ${ }^{a}$ S. Duarte Pinto, ${ }^{a, c}$ L. Ropelewski, ${ }^{a}$ F. Sauli, ${ }^{a, d}$ M. Villa ${ }^{a, c}$ and E. Rocco ${ }^{e}$}

\author{
${ }^{a}$ CERN, Geneva, Switzerland \\ ${ }^{b}$ University of Siena \& INFN Pisa, Siena, Italy \\ ${ }^{c}$ University of Bonn, Bonn, Germany \\ ${ }^{d}$ TERA Foundation, Novara, Italy \\ ${ }^{e}$ University of Eastern Piedmont \& INFN Torino, Alessandria, Italy \\ E-mail: gabriele.croci@cern.ch
}

AbSTRACT: A voltage-controlled Gas Electron Multiplier (GEM) can be used to block the reinjection of positive ions in large volume Time Projection Chambers (TPC). Through an accurate choice of proper geometry, gas filling and external fields it is possible to obtain a sufficient level of electron transmission at very low GEM voltages (Gating GEM) despite the degradation of energy resolution due to the loss of primary electrons. The addition of a pre-amplification GEM in front of the Gating GEM causes an improvement in energy resolution while keeping the ion feedback at the level of primary ionization. The measurements show that a small pulse of about $40 \mathrm{~V}$ completely closes the gate, stopping the ions produced in the amplification stage.

KEYWORDS: Gaseous detectors; Micropattern gaseous detectors (MSGC, GEM, THGEM, RETHGEM, MICROMEGAS, InGrid, etc)

\footnotetext{
${ }^{1}$ Corresponding author.
} 


\section{Contents}

1 Introduction 1

2 The small TPC prototype 2

2.1 Gating GEM transparency measurements 2

2.2 Energy resolution improvement 3

2.3 Gating GEM voltage scan with preamplifier GEM 5

2.4 Preamplification GEM Ion back flow measurement 6

$\begin{array}{lll}2.5 & \text { Amplification stage voltage scan } & 6\end{array}$

2.6 Full detector behaviour $\quad 7$

3 Conclusions $\quad 8$

\section{Introduction}

The Gas Electron Multiplier (GEM) [2] , consists of a thin Kapton insulating foil copper-clad on both sides and perforated by a high density, regular matrix of holes (50 to 100 per square millimetre). The distance between holes (pitch) is typically $140 \mu \mathrm{m}$ and the diameter is of about $70 \mu \mathrm{m}$. Upon an application of a potential difference between the GEM electrodes, a high dipole field develops in the holes, focusing the field lines between the drift electrode and the readout element. Electrons drift along the channel and the charge is amplified by a factor that depends on the field intensity and on the length of the channel.

Large volume gaseous Time Projection Chambers (TPCs) suffer from the problem of ionbuilt space charge. The amplification structures (nowadays still MPWC) amplifies the primary electrons created by the interacting particle and consequently generates ions that slowly drift back to the drift volume where they modify the electric field thus changing the time-space properties of the next track. Next generation TPC endcap will maybe be equipped with Micro Pattern Gas Detector (MPGD) such as GEM and Micromegas ([4-6]). Gating electrodes are needed to prevent the re-injection of positive ions into the large gas volume and during LEP (and LHC) period the wire-gating technique was extensively used. The next generation gating structures can be made by MPGDs. When a GEM foil is powered at very low potential difference (from $10 \mathrm{~V}$ up to 40V) [1] it does not act as an electron amplifier device. Its electron transparency (the ratio between the number of electrons that are able to pass trough the GEM holes and the numbre of electrons present in front of the top GEM electrode) is reduced to few tens of percent depending on the applied potential difference, on the external fields, on the GEM geometry and on the chosen filling gas. A voltagecontrolled Gas Electron Multiplier (GEM) powered at low potential difference can be used to block the re-injection of positive ions in large volume Time Projection Chambers. A gated pulse that inverts the GEM potential difference stops all the ions produced in the amplification stages below the gating GEM. In this paper we investigated the basic physics processes under this application but real pulse gating will be matter of future work. 


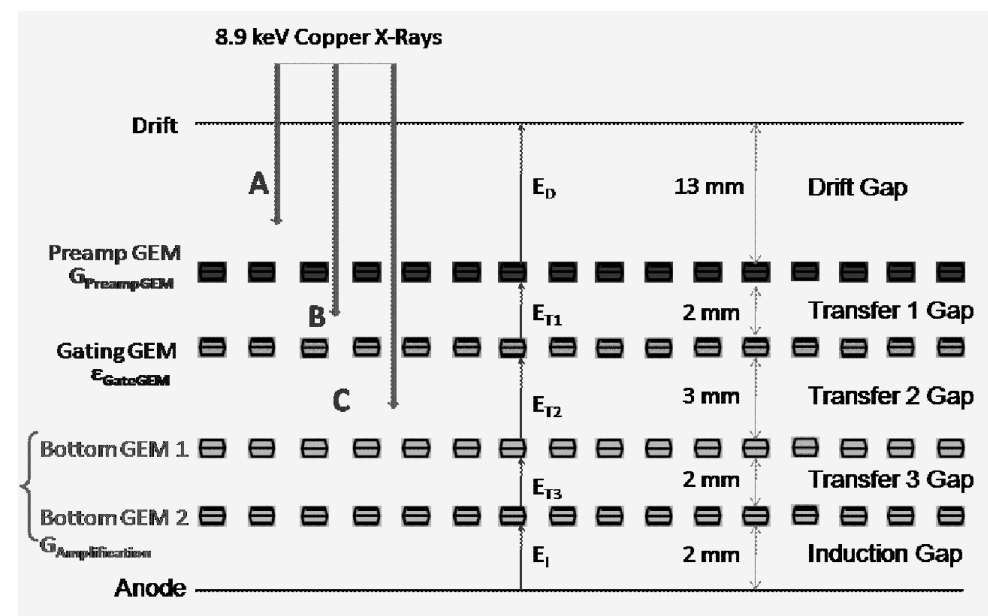

Figure 1. Small prototype schematics. The readout anode was a full metal plane

\section{The small TPC prototype}

Figure 1 shows the small TPC scheme. Two different configurations were studied: in the first one the first GEM foil was used as Gating GEM; in the second one another GEM (Preamplification GEM) foil was added in front of the Gating GEM in order to get a preamplification factor. The final amplification stage was represented by a double GEM structure (Bottom GEMs). All the GEM foils used for the measurements are standard GEM foils (50 $\mu \mathrm{m}$ hole diameter, $140 \mu \mathrm{m}$ pitch); the drift cathode was a metallized mylar plate and the anode was a full copper plane. The pulse height spectra were acquired using an ORTEC 142-IH preamplifier and an ORTEC 450 research amplifier; the current was read-out by means of a Keithley 6517A picoamperometer ( $\sim 1 \mathrm{pA}$ resolution). The detector performance was studied by means of a collimated $8.9 \mathrm{keV}$ X-Rays beam impinging either orthogonally or parellely to the drift cathode. When the direction was orthogonal to the drift cathode, the X-Rays could convert in three different detector gaps: in the drift gap (A region), in the transfer 1 gap ( $B$ region) or in the transfer 2 gap $(C$ region); when the direction was parallel to the drift cathode, the conversions happened only in the drift gap (A region, see figure 8 ). The gas mixture used in all the characterization was $\mathrm{Ar} / \mathrm{CO}_{2} 70 \% / 30 \%$.

\subsection{Gating GEM transparency measurements}

When a low potential difference (from $5 \mathrm{~V}$ to $50 \mathrm{~V}$ ) is applied to a GEM foil [1], it does not act as an amplification device but rather it absorbs part of the charge that approaches it and its electron transparency ( $\varepsilon_{\text {GatingGEM }}$, the ratio between outcoming and incoming electrons) is not $100 \%$ but depends on the $\triangle \mathrm{V}_{G E M}$ and on the external electric fields [3]. Figure 2 shows that the measured electron transparency has a value around $30 \%$ for $10 \mathrm{~V} \leq \Delta V \leq 40 \mathrm{~V}$. The Gating GEM electron transparency was obtained by the ratio between the $B$ pulse height peak position and the $C$ pulse height peak position (see figure 3). The energy resolution is worsened by the presence of the Gating GEM (from 20\% FWHM up to 60\% FWHM) and this effect is clearly visible in figure 3, that shows the pulse hight spectra acquired for different $\Delta \mathrm{V}_{\text {GatingGEM }}$. 


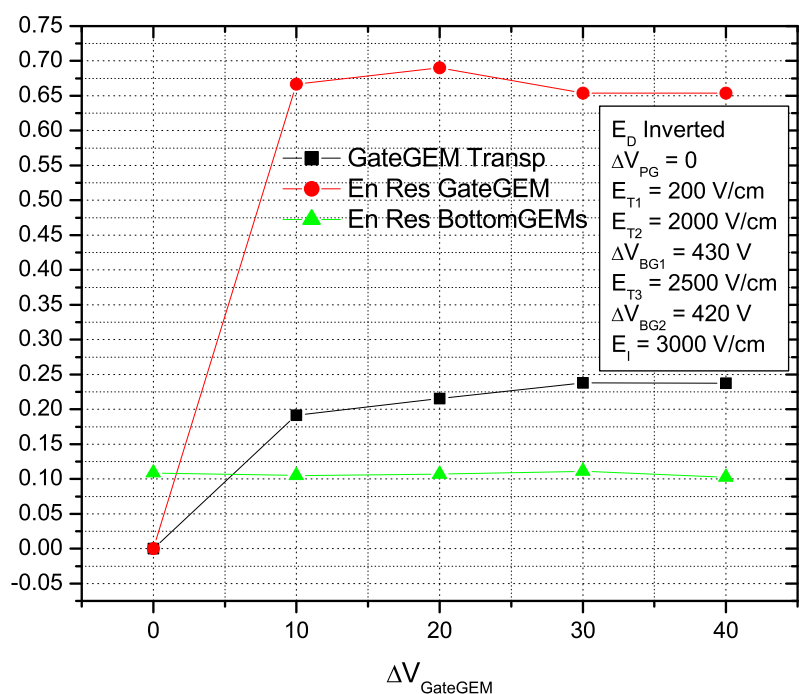

Figure 2. Gating GEM electron transparency measurements; the plot for Bottom GEMs represents the energy resolution obtained for X-Rays conversions under the Gating GEM (C region); all energy resolutions are FWHM

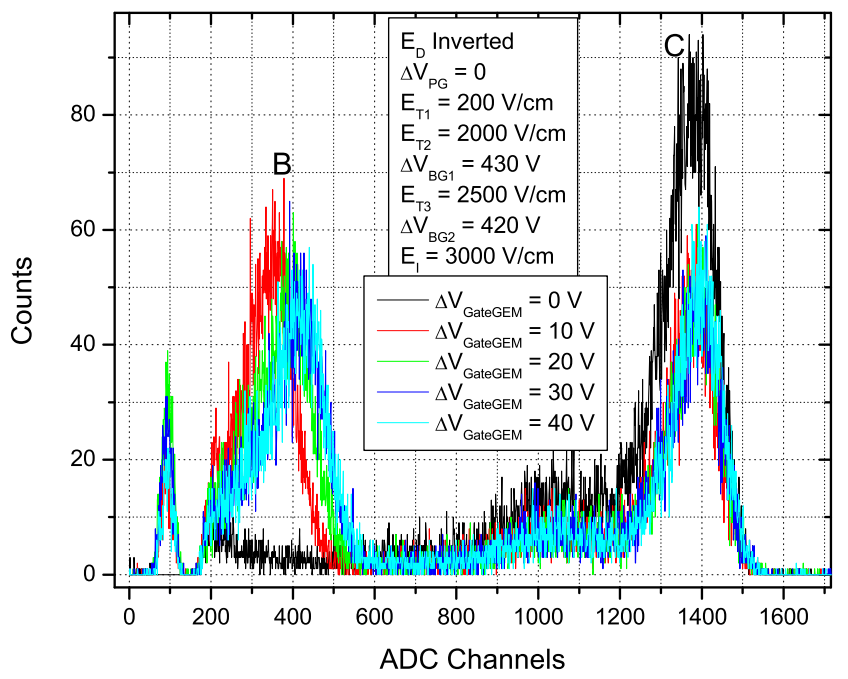

Figure 3. Pulse Height spectra to measure Gating GEM electron transparency: only the peaks coming from conversion in $B$ region and $C$ region are visible beacause the drift field was inverted. The leftmost peak is the electronic pedestal

\subsection{Energy resolution improvement}

The use of a low-voltage GEM as first electrode causes a degradation of the energy resolution because part of the primary electrons are absorbed in the top electrode of the GEM itself. In order 


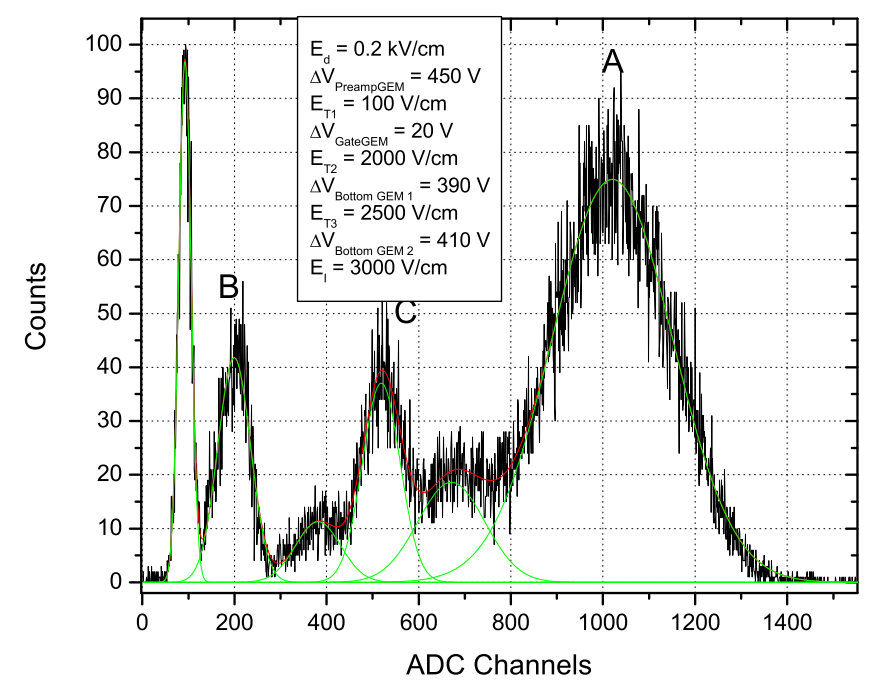

Figure 4. Typical PH spectra acquired with preamplification on. The $B, C, A$ regions peaks are well visible

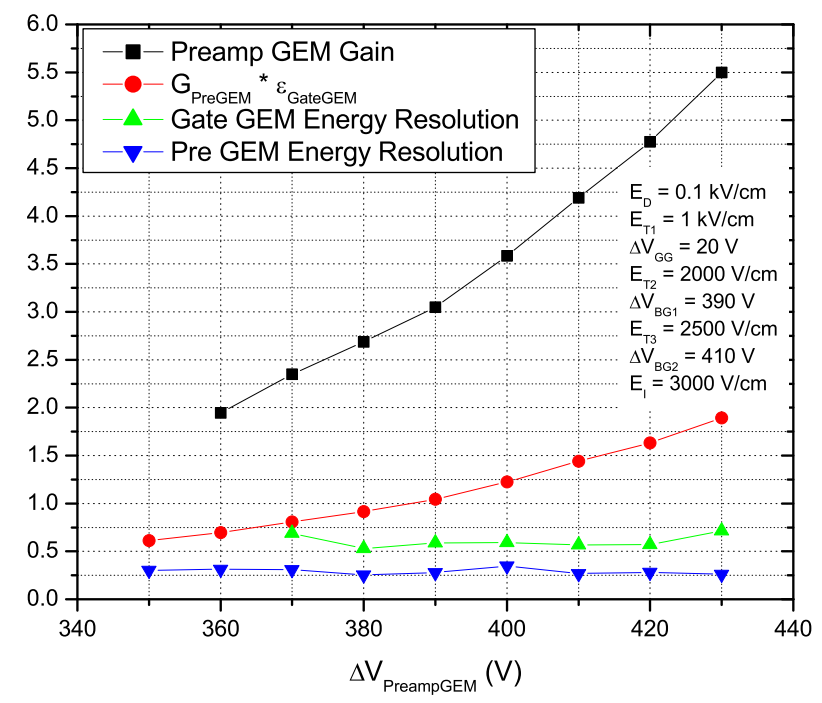

Figure 5. $\Delta V_{\text {PreampGEM }}$ scan: the red circles represent the $G_{F i r s t S t a g e}$, the up-green (down-blue) side triangles the energy resolution (FWHM) for $\mathrm{B}(\mathrm{C})$ peaks

to get an improvement of energy resolution, another GEM foil (Preamplification GEM) was added before the Gating GEM. This is an useful method to preamplify primary electrons before loosing part of them in the gate electrode. The typical PH spectra obtained when the Preamplification GEM was operational is shown in figure 4.

As it is shown in figure 5, the $\Delta V$ applied on the Preamplification GEM varies between $350 \mathrm{~V}$ and $430 \mathrm{~V}$, giving a gain (measured as $\left.G_{\text {PreamplificationGEM }}=\frac{A_{\text {PHPeakPsition }}}{B_{P H P e a k P s i t i o n}}\right)$ between 2 and 5.5. This gain was also obtained through the optimization of external $\left(E_{T 1}, E_{T 2}\right)$ electric fields.

The aim of this exercise was to get a unitary first stage gain $\left(G_{\text {FirstStage }}=G_{\text {PreampGEM }} *\right.$ 


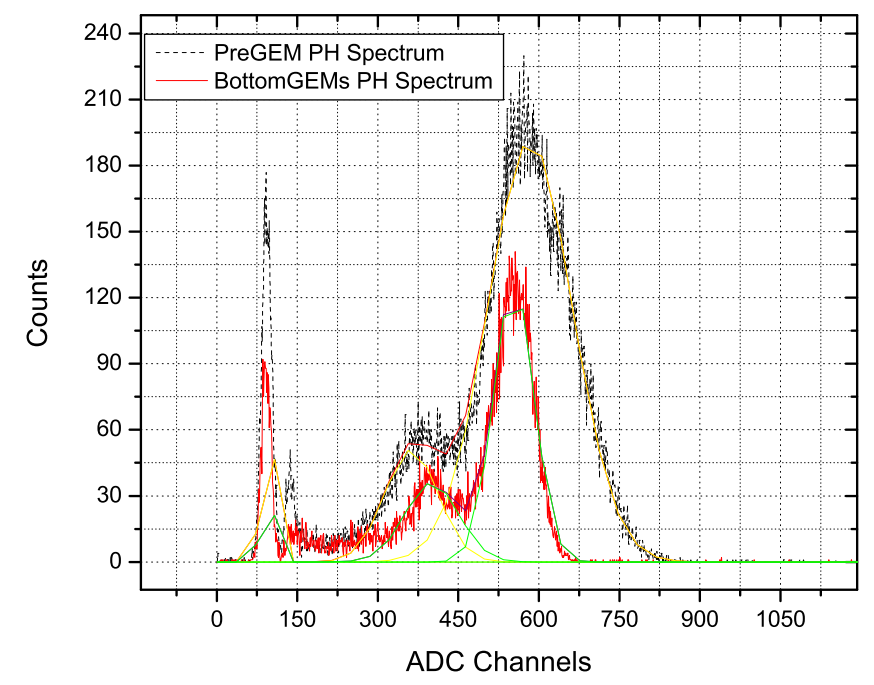

Figure 6. First stage unitary gain: the $C$ (red) and $A$ (black) peaks overlap (the $C$ peak was obtained with $\Delta V_{\text {Gating GEM }}=-20$ )

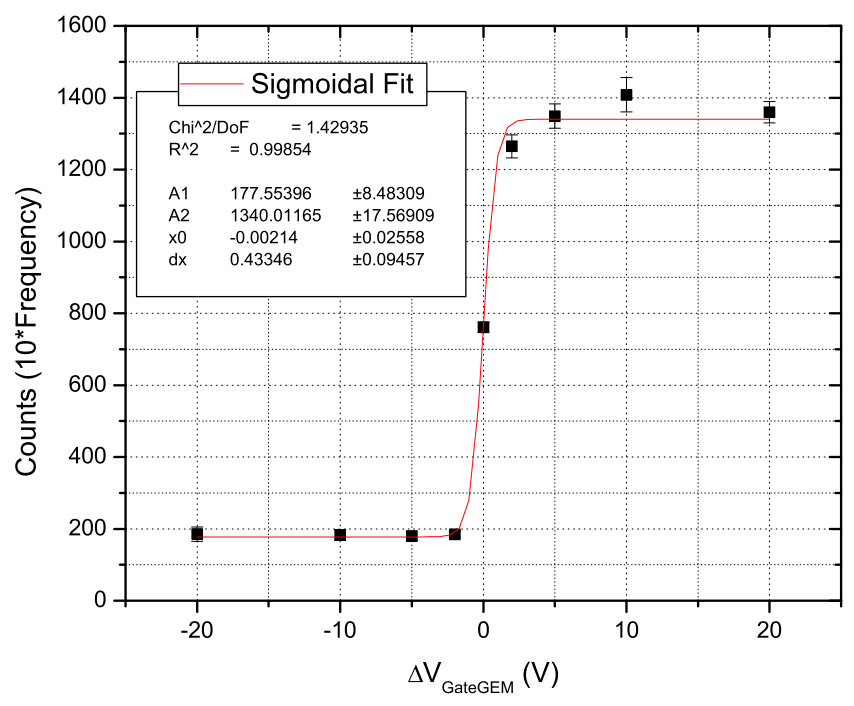

Figure 7. $\Delta V_{\text {GateGEM }}$ scan with Preamplification GEM on. Negative potential sign means closed gate

$\varepsilon_{\text {GatingGEM }}$ ) by adjusting $\triangle V_{\text {PreampGEM }}$. As it is shown in figure 6 this result was obtained with $\Delta V_{\text {PreampGEM }}=390 \mathrm{~V}$. It was also proven that the addition of the Preamplification GEM (figure 5) improved the energy resolution by a factor of 2, from 60\% FWHM down to 30\% FWHM.

\subsection{Gating GEM voltage scan with preamplifier GEM}

In order to prove the gating properties of the Gating GEM, a counting mode $\Delta V_{\text {GatingGEM }}$ scan was made and the corresponding photon interaction rate was recorded. As it is shown in figure 7 the potential difference applied to the Gating GEM varied from $-20 \mathrm{~V}$ up to $+20 \mathrm{~V}$ (where the positive 


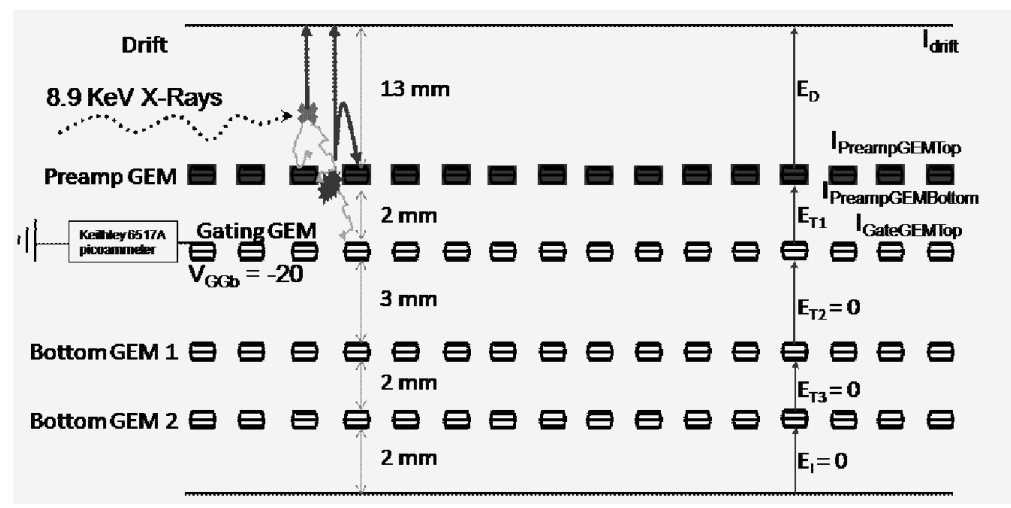

Figure 8. Detector Scheme to measure Preamplification GEM Normalized Ion Feedback

sign means correct polarization to get electrons drifting in the holes): this step demonstrated that the gate is completely closed with a very small potential difference, from $-10 \mathrm{~V}$ to $-5 \mathrm{~V}$. This is a very promising feature for high rate pulsed gate in TPCs. The closed gate plateau is not on the zero level because of X-Rays conversion in the $C$ region.

\subsection{Preamplification GEM Ion back flow measurement}

In a real TPC operation all the ions produced in the amplification stages below the gate will be stopped by closing the gate. Being the Preamplification GEM placed above the gate, it is crucial to understand how many of the ions produced in this stage will drift back to the large gas volume. The setup used to measure the Preamplification GEM ion feedback is shown in figure 8: only the very top part of the prototype was used and irradiated by an X-Rays beam parallel to the drift cathode. In this way only conversions in the drift gap were obtained and currents on drift $\left(I_{\text {Drift }}\right)$, PreampGEM top electrode ( $\left.I_{\text {PreampGEMTop }}\right)$, Preamp bottom electrode $\left(I_{\text {PreampGEMBottom }}\right)$ and GateGEM top electrode $\left(I_{\text {GatingGEMTop }}\right)$ were measured. Knowing from previous measurements the value of the ionization current (IIonization), it was possible to define the (PreampGEM) Normalized Ion Feedback, $N I F$ as NIF $=\frac{I_{\text {drift }}}{I_{\text {ionization }}}$. This definition is related to the Common Ion Feedback definition $(C I F)$ as

$$
C I F=\frac{I_{\text {drift }}}{I_{\text {readout }}}=\frac{\text { NIF }}{\text { Gain }_{\text {FullDetector }}}
$$

Figure 9 shows that in the scanned Preamplification GEM voltage range with a fixed drift field $\left(E_{d}\right)$ value of $0.1 \mathrm{kV} / \mathrm{cm}$, a NIF around 2-3 was measured, the same order of magnitude of primary ionization current. The conclusion is that the ion feedback contribution introduced by the preamplification stage is not much higher than the primary ionization itself.

\subsection{Amplification stage voltage scan}

The experimental setup shown in figure 8 was used in order to understand if the Gating GEM was working independently from the amplification stage. The measurement of the NIF was performed changing the potential difference on one of the two amplification GEM $\left(\triangle V_{\text {BottomGEM2 }}\right)$, keeping the same $\Delta V_{\text {PreampGEM }}=390 \mathrm{~V}$ and closing the gate $\left(\Delta V_{\text {GatingGEM }}=-20 \mathrm{~V}\right)$. Figure 10 shows that 


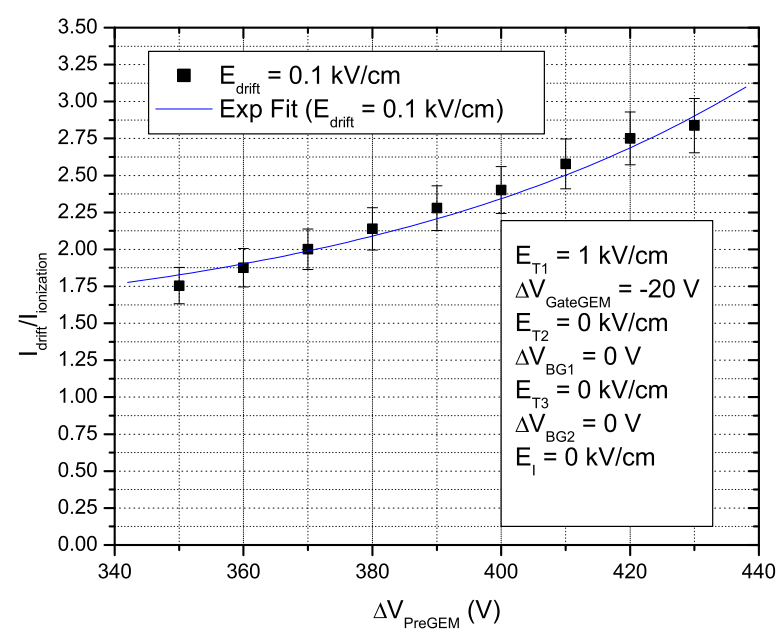

Figure 9. Normalize Ion Feedback for $350 \mathrm{~V} \leq \Delta V_{\text {PreAmp } G E M} \leq 440 \mathrm{~V}$

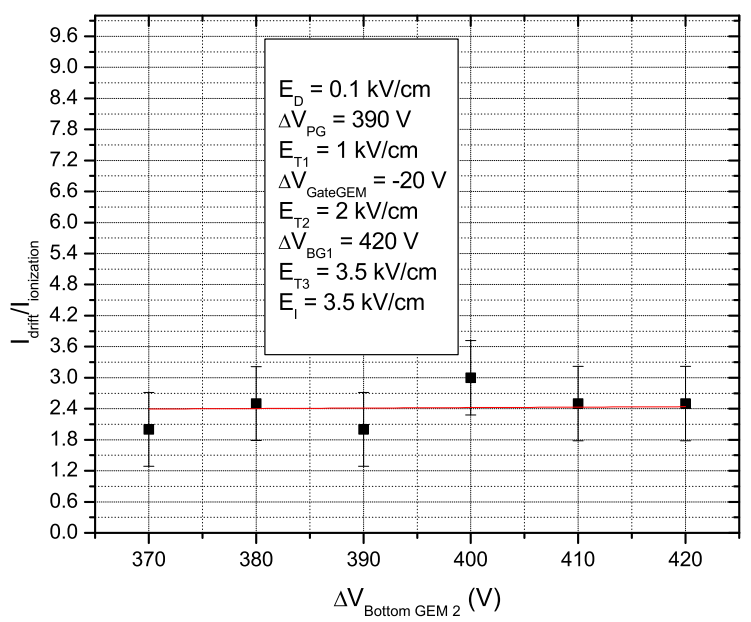

Figure 10. Normalize Ion Feedback for $370 \mathrm{~V} \leq \Delta V_{\text {BottomGEM } 2} \leq 420 \mathrm{~V}$

the measured NIF corresponds to a value of 2-3: this number underlines that the NIF is only due to the Preamplification GEM.

If the gate is opened $\left(\Delta V_{\text {GatingGEM }}=+20 \mathrm{~V}\right)$ the point $@ \Delta V_{\text {BottomGEM2 }}=400 \mathrm{~V}$ will correspond to a Gain $_{\text {FullDetector of about } 3000 .}$

\subsection{Full detector behaviour}

Figure 11 shows a $\Delta V_{\text {GatingGEM }}$ scan that summarizes the prototype behaviour.

When the gate is completely closed $\left(\Delta V_{\text {GatingGEM }}=-20 \mathrm{~V}\right)$ the overall gain $\left(\frac{I_{\text {readout }}}{I_{\text {ionization }}}\right)$ is zero (no electron can get to the amplification stage) and the NIF is 2-3 and this corresponds to the PreampGEM NIF. When the gate is open $\left(\Delta V_{\text {Gating } G E M}=+20 \mathrm{~V}\right)$ the overall gain is around 3000 but it can be increased if one of the two amplification GEM voltages is increased. 


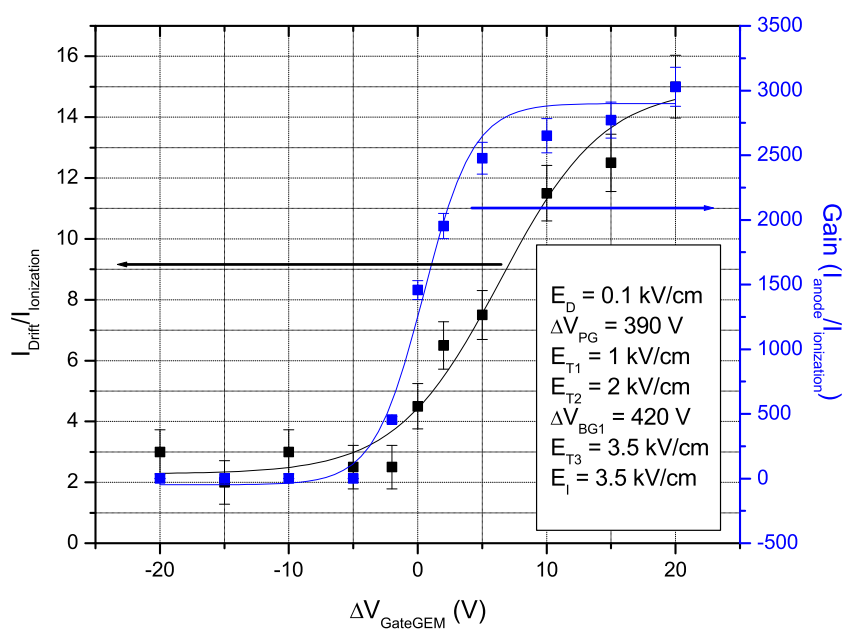

Figure 11. Normalize Ion Feedback (black curve - left y-axis) and Overall Gain (blue curve - right y-axis) vs $\Delta V_{\text {GatingGEM }}$

\section{Conclusions}

The standard GEM foil can be used as a gate electrode for a TPC when a low potential difference is applied to its electrodes. A small pulse (20V-40V) is sufficient to open and close the gate, giving the possibility to have a very high rate pulsed gating. The measurements prove also that the energy resolution is improved by the addition of a properly operated Preaplification GEM in front of the Gating GEM and that the Preamplification GEM does not largely contribute to the Normalized Ion Feedback, NIF (Preamp GEM NIF = 2-3). In addition, since all the ions produced in the last stage are stopped by the gate, the amplification stage gain can be as high as required by the experiment.

\section{References}

[1] F. Sauli, L. Ropelewski and P. Everaerts, Ion feedback suppression in time projection chambers, Nucl. Instrum. Meth. A 560 (2006) 269.

[2] F. Sauli, GEM: a new concept for electron amplification in gas detectors, Nucl. Instrum. Meth. A 386 (1997) 531.

[3] S. Bachmann et al., Charge amplification and transfer processes in the gas electron multiplier, Nucl. Instrum. Meth. A 438 (1999) 376.

[4] Y. Li et al., Performance study of a GEM-TPC prototype using cosmic rays, Nucl. Instrum. Meth. A 596 (2008) 305.

[5] M. Killenberg et al., Development of a GEM-based high resolution TPC for the International Linear Collider, Nucl. Instrum. Meth. A 573 (2007) 183.

[6] S. Anvar et al., Large bulk MicroMegas detectors for TPC applications, Nucl. Instrum. Meth. A 602 (2009) 415. 